\title{
Studies on Electric field strength distribution of uhf television signal propagation in Ekiti State. Nigeria.
}

\author{
Olumuyiwa Oludare FAGBOHUN \\ Department of Electrical \& Electronic Engineering, Ekiti State University, PMB 5363, Ado-Ekiti, Nigeria.
}

\begin{abstract}
Researchers have continued to carry-out work to determine the actual coverage area of some broadcasting stations and the level of signal strength variation with distance from the transmitter, which is useful for radio transmission designs.In Nigeria, few works exist on the frequency modulation (FM) signals propagation, and little work has been done on the television signals. The work determines the actual coverage of the Broadcasting Service of Ekiti State Television, in Ekiti - State, southwest Nigeria, with the expected coverage area being the entire land mass of the State. The method used is such that requires the physical presence of the investigator in all the towns and villages within the State with the necessary instrument to carry out the electric field strength measurements in all the locations. The variation of signal level with distance (i.e. Propagation curve) was analysed, to reveal areas of optimum coverage and areas that are poorly serviced.The coverage of the newly installed transmitter in the station was compared to the old transmitter (which had been in use for over 30 years), and a comprehensive statistical data base provided for the UHF television broadcasting Station. The results shows the field strength distribution of the broadcasting station in major towns and villages,its elevation pattern and the various grades of coverage enjoyed by its citizens in such locations. Recommendations were made on getting areas outside optimum coverage well serviced.
\end{abstract}

Keywords: Coverage area, Reception, Signal strength variations, Transmission,Ultra high frequency.

\section{Introduction}

In view of the importance of television broadcasting to the socio-economic development of the populace and the competition in the business, viewers interest has grown from just watching any thing on screen to qualitative, clean and sharp signals on television screen. All stations have their expected coverage area and their signals should not constitute interference to others [1]. The Broadcating Service of Ekiti State (BSES), is the electronic media outfit of the government of Ekiti State, Southwest, Nigeria. It is a combination of both the radio and television services. The television service is on channel 41 on the ultra high frequency band while, the radio services operates on $91.5 \mathrm{MHz}$ and $532 \mathrm{kHz}$ for the FM and AM radios respectively. The BSES Ch 41 UHF (631.25MHz carrier frequency) is located at broadcasting house, Ilokun quarters, Ado Ekiti, the State capital, with a staff strength of 289. The station provides services in the areas of education, information, entertainment and social mobilization. The televisions works 15 hours daily [2]. The coverage area for broadcasting channels is the radial distance away from the transmitter in which the transmited signal, voice and picture for television and voice for radio can be received by the viewer or listeners with the aid of a receiving antenna.These are classified into three, namely: i). Primary Coverage Areadefined as a region about the transmitting station in which the signal strength is strong enough to override ordinary interference at all times, with ahigh level of dependancy and clarity, with or without the use of an active antenna. ii). Secondary Coverage Area being a region about the transmitting station, with the signal strength often sufficient to be useful but not strong enough to overried ordinary interference at all times, and requires an active receiving antennafor a clear reception. iii). Fringe/Tertiary Coverage Area is a region about the transmitting station, where the signal strength is weak and not dependable, with services neither guaranteed nor protected against interference, and where the use of an active receiving antenna may not bring about a clear reception at all times[3,4].

Free space or the earth's atmosphere is often used as a transmission medium for wave propagation $[5,6]$. To propagate transverse electromagnetic waves through the earth's atmosphere, it is necessary that the energy be radiated from the source (Base station) and then the energy captured at the receiving end which are functions of antennas, with radiating energy antenna called the transmitter and the capturing energy antenna called the receiver [6,7].Space wave propagation includes radiated energy that travels in the lower few miles of the earth atmosphere, and include both direct and ground - reflected waves. Direct waves travel essentially in a straight line between the transmit and receive antennas commonly called line - of - sight (LOS) transmission, while ground reflected waves are those waves that are reflected by the earths surface as they propagate between the transmit and receiver antennas. Field intensity at the receive antenna depends on the distance between the two antenna (attenuation and absorption) and whether the direct and ground reflected waves are in phase (interference) $[7,8]$. The curvature of the earth presents a horizon to space wave propagation called the radio 
horizon, and due to atmospheric refraction, the radio horizon extends beyond the optical horizon for the common standard atmosphere[6,8]. The radio horizon is approximately four - thirds that of the optical horizon. Refraction is caused by the troposphere due to changes in its density, temprature, water vapour content, and relative conductivity, and radio horizon can be lengthened simply by elevating the transmit or receive antennas above the earth's surface with towers or by placing them on top of mountains or high buildings $[8,9,10]$.The strength of the electric field $\mathrm{E}$ (in volts/meter) at a distance $\mathrm{r}$ from a point source is given byE $=30 \mathrm{P}_{\mathrm{t}} / \mathrm{r}$; where $P_{t}$ is the original transmitted power in watts, and one of the maxwell's equations [6,7,10]. Effective isotropic radiated power (EIRP) is defined as an equivalent transmit power and is expressed mathematically as

$$
\mathrm{EIRP}=\mathrm{P}_{\mathrm{r}} \mathrm{A}_{\mathrm{t}} \text { watts }
$$

Where $P_{\mathrm{r}}=$ total radiated power, $\mathrm{A}_{\mathrm{t}}=$ transmit antenna directive gain or

$$
\operatorname{EIRP}(\mathrm{dBm})=10 \log \underline{P_{\mathrm{r}}}+10 \log \mathrm{A}_{\mathrm{t}}
$$

EIRP or simply ERP (effective radiated power) is the equivalent power that an isotropic antenna would have to radiate to achieve the same power density in the chosen direction at a given point as another antenna. The power density ata given point, when expanded to include the transmit antenna gain is

$$
\mathfrak{J}=\frac{P_{r}}{4} \frac{A_{t}}{R^{2}}
$$

Antennas are reciprocal devices; an antenna has the same power gain and directivity when it is used to receive electromagnetic waves as it has for transmitting electromagnetic waves. Consequently, the power received or captured by an antenna is the product of the power density in the space immediately surrounding the antenna and the antenna directive gain. Therefore,

captured power $=\mathrm{c}=\mathfrak{I}_{\mathrm{r}}=\underline{\mathrm{P}}_{\mathrm{r}} \cdot \mathrm{A}_{t} \frac{\mathrm{A}_{\mathrm{r}}}{4 \pi \mathrm{R}^{2}}$

Where $A_{r}$ is the receiver antenna power gain. The captured power is not all useful; some of it is dissipated in the receive antenna [7,10]. The actual useful received power is the product of the received power density, the receive antenna's direct gain, and the receive antenna's efficiency or the receive power density times the receive antenna's power gain. If an antenna is lossless, it radiates $100 \%$ of the input power and the power gain is equal to the directive gain $[8,11,13]$. An antenna does not actually amplify the input power, but concentrates its radiated power in a particular direction. If gain is realized in one direction, a corresponding reduction in power density (a loss) must be realized in another direction [11,13]. The direction in which an antenna is pointing is always the direction of maximum radiation, and for maximum radiation, and maximum captured power, a receiver antenna must be pointing in the direction from which the reception is desired. Table 1 shows the radio frequency spectrum with UHF of frequency band $300 \mathrm{MHz}$ to $3 \mathrm{GHz}$ used for television broadcasting.

Table 1: Radio Frequency Spectrum and Uses [2]

\begin{tabular}{|l|l|l|}
\hline Frequency Band & Descriptive Designation & Uses \\
\hline $3-30 \mathrm{kHz}$ & Very Low Frequency (VLF) & In radio navigation system \\
\hline $30-300 \mathrm{kHz}$ & Low Frequency (LF) & In submarine link \\
\hline $300-3000 \mathrm{kHz}$ & Medium Frequency & AM Radio Broadcast, Links for ships \\
\hline $3-30 \mathrm{MHz}$ & High Frequency (HF) & Broadcasting; radio link satellite communication \\
\hline $30-300 \mathrm{MHz}$ & Very High Frequency (VHF) & TV Broadcasting, point to point communication \\
\hline $300-3000 \mathrm{MHz}$ & Ultra High Frequency (UHF) & TV Broadcasting, RADAR, Radio Navigation \\
\hline $3-30 \mathrm{GHz}$ & Super High Frequency (SHF) & Satellite communications, RADAR, Radio Navigation \\
\hline $30-300 \mathrm{GHz}$ & Extremely High Frequency (EHF) & For Research \\
\hline
\end{tabular}

\section{Materials and Methods}

2.1 Materials used: The following instruments and materials were used in the course of carrying out this research;

i. The Dagatron Tm 10 Level (field strength) Meter, with frequency range $(5 \mathrm{MHz}-862 \mathrm{MHz})$, measurement range $(15-120 \mathrm{~dB} \mu \mathrm{V})$, resolution $(0.1 \mathrm{~dB})$, and accuracy $(+/-3 \mathrm{~dB})$, and Unit of measurement in $\mathrm{dB}-\mu \mathrm{V}$.

ii. The Global Positioning System (GPS) receiver; GARMIN MAP 76 Personal Navigator

iii. TV receiving antenna(passive), I-conductor and coaxial cable and,

iv. Ekiti State Administrative map. 


\subsection{Research Methodology}

The Electric Field Strength measurements were conducted on channel 41 ultra-high frequency (UHF) television signal of the Broadcasting Service of Ekiti State (BSES). As at the time of carrying out this work, only the Broadcasting Service of Ekiti State (BSES) transmits on the UHF band in the state. The transmitting station has a transmitter maximum output capacity of $40 \mathrm{~kW}$. However, the transmitted power by the station was $20 \%$ of the output capacity.This station is located at Ilokun quarters, Ado Ekiti, in the state capital with transmitter coordinate (Lat. $7.71^{\circ} \mathrm{N}$, Long. $5.21^{0} \mathrm{E}$ ). It has two transmitters which allows one to be on standby, while the other is on air. This transmitter is the solid state type except the power amplifier (PA) stage. The PA stage makes use of the inductive Oxide Tube Technology (IOT) which is an improved klystron technology. However, the newly installed transmitter that began transmission in February 2011 was a complete solid state type.

The Electric Field Strength measurements of this transmitting station were carried out radially along different routes from the base station (as reference). These measurements were taken using the digital field strength meter (covering the frequency range $5.0-862.0 \mathrm{MHz}$ and measurement range $15.0-12 \mathrm{~dB} \mu \mathrm{V}$ ). The GPS receiver (GARMIN MAP 76) model, personal navigator was used to monitor the line of sight of the different locations where data was collected from the base station. It was also used to determine the latitude, longitude and the elevation above sea level of the locations. Measurements were taken at interval of $5 \mathrm{~km}$ in the major axes from the base station, and in majority of the towns and villages in all the local government areas visited as far as good road accessibility permitted and except in areas where the signals had faded away. These measurements were carried out in the months of June and July 2010. Arrangements were made with the station to ensure continuous operation of the transmitter during the data collation period. Transmission parameters were monitored and found to be substantially constant throughout this period.The second phase of survey (measurements) was conducted on the station in July 2011 with the primary objective of comparing the coverage of the new transmitter to the old one. The transmitted power of the new transmitter during this work was $20 \mathrm{~kW}$. The need to wait and take the measurement in July was to ensure uniformity in the month of measurement with the first phase. Eight local government areas were used as samples. The electric field strength of the transmitted television signal for different locations with their corresponding distances (LOS) from the base station were recorded. Also, determined and recorded were the latitude, longitude and the altitude of the various locations where data were collected.

Table 2: Transmission Parameter for Ch.41 TV. Station at Ado Ekiti for the First Phase of Measurement in the Months of June and July 2010.

\begin{tabular}{|l|l|l|}
\hline S/N & PARAMETER & VALUE FOR ROUTES A,B \\
\hline 1 & Base station transmitting power & $8000 \mathrm{~W}(20 \%$ of $40 \mathrm{~kW})$ \\
\hline 2 & Base station carrier frequency & $631.25 \mathrm{MHz}$ \\
\hline 3 & Transmitter in use & Harris $40 \mathrm{~kW}$ \\
\hline 4 & Height of transmitting mast & $200.0 \mathrm{~m}$ \\
\hline 5 & Height of transmitting antenna & $18.29 \mathrm{~m}(60 \mathrm{ft})$ \\
\hline 6 & Transmitting antenna gain & $32 \mathrm{~dB}$ \\
\hline 7 & Height of receiving antenna & $1.94 \mathrm{~m}(6 \mathrm{ft})$ \\
\hline
\end{tabular}

Table 3: Transmission Parameter for Ch.41 TV. Station at Ado Ekiti for the Second Phase of Data Collection in November 2011

\begin{tabular}{|l|l|l|}
\hline S/N & PARAMETER & VALUE FOR ROUTES A,B \\
\hline 1 & Base station transmitting power & $20 \mathrm{~kW}$ \\
\hline 2 & Base station carrier frequency & $631.25 \mathrm{MHz}$ \\
\hline 3 & Transmitter in use & Harris maxima solid state type \\
\hline 4 & Height of transmitting mast & $200.0 \mathrm{~m}$ \\
\hline 5 & Height of transmitting antenna & $13 \mathrm{~m}$ \\
\hline 6 & Transmitting antenna gain & $13 \mathrm{~dB}$ \\
\hline 7 & Height of receiving antenna & $1.94 \mathrm{~m}(6 \mathrm{ft})$ \\
\hline
\end{tabular}


Studies on Electric field strength distribution of uhf television signal propagation in Ekiti State. Nigeria.

\section{Results and Discussion}

\subsection{Field Strength Measurement}

The electric field strength data collected in the state with some communication parameters taken at data location points with the old transmitter in the months of June and July 2010 are as tabulated in Table 3 and 4. With Table 5 showings the transmission parameters.

Table 4: Result of the Electric Field Strength Measurement of Channel 41UHF Television Signal (631.25MHz), of the Broadcasting Service of Ekiti State (BSES) with the old transmitter: Data collected in the months of June and July 2010. Route A.

\begin{tabular}{|c|c|c|c|c|c|c|c|c|}
\hline $\begin{array}{l}\text { Observati } \\
\text { on }\end{array}$ & $\begin{array}{l}\text { Line of } \\
\text { sight from } \\
\text { the } T X \\
\text { base }(\mathbf{k m})\end{array}$ & $\operatorname{Lat}\left({ }^{\circ} \mathbf{N}\right)$ & Long $\left({ }^{\circ} \mathbf{E}\right)$ & $\begin{array}{l}\text { Elevation } \\
\text { ASL/AGL } \\
\text { (m) }\end{array}$ & $\begin{array}{l}\text { Multiple } \\
\text { reading } \\
\text { /E/ field } \\
\text { strength } \\
(\mathrm{dB} \mu \mathrm{V})\end{array}$ & $\begin{array}{l}\text { Average } \\
\text { reading } \\
/ \mathrm{E} / \\
(\mathrm{dB} \boldsymbol{\mu} \mathrm{V})\end{array}$ & $\begin{array}{l}\text { Description of } \\
\text { location/Town }\end{array}$ & $\begin{array}{l}\text { Local } \\
\text { Govern } \\
\text { ment } \\
\text { Area. } \\
\text { (LGA) }\end{array}$ \\
\hline 1 & 0.00 & 7.72582 & 5.2356 & 415.22 & $\begin{array}{l}80.52 \\
81.22 \\
80.32\end{array}$ & 80.687 & $\begin{array}{l}\text { Base } \\
\text { transmitting } \\
\text { antenna at } \\
\text { Ekiti. }\end{array}$ & Ado \\
\hline 2 & 5.02 & 7.191848 & 5.27946 & 409.12 & $\begin{array}{l}66.12 \\
67.82 \\
66.92\end{array}$ & 66.413 & UNAD, Ekiti & Ado \\
\hline 3 & 4.98 & 7.73537 & 5.26879 & 418.42 & $\begin{array}{l}63.52 \\
64.92 \\
64.52\end{array}$ & 64.320 & UNAD 2 Campus & Ado \\
\hline 4 & 6.36 & 7.75046 & 5.28120 & 432.12 & $\begin{array}{l}69.02 \\
68.92 \\
69.22 \\
\end{array}$ & 69.053 & IworokoEkiti & $\begin{array}{l}\text { Irepodun/ } \\
\text { Ifelodun }\end{array}$ \\
\hline 5 & 10.04 & 7.78520 & 5.27211 & 562.32 & $\begin{array}{l}44.52 \\
45.82 \\
45.12 \\
\end{array}$ & 45.153 & Near IfakiEkiti & IdoOsi \\
\hline 6 & 12.61 & 7.80841 & 5.26291 & 571.32 & $\begin{array}{l}46.52 \\
47.52 \\
47.92 \\
\end{array}$ & 47.320 & IfakiEkiti & IdoOsi \\
\hline 7 & 14.00 & 7.82055 & 5.25759 & 574.42 & $\begin{array}{l}31.62 \\
37.62 \\
38.52\end{array}$ & 34.920 & IfakiEkiti & IdoOsi \\
\hline 8 & 17.55 & 7.85246 & 5.25486 & 563.62 & $\begin{array}{l}26.02 \\
27.52 \\
26.72 \\
\end{array}$ & 26.770 & Orin Ekiti & IdoOsi \\
\hline 9 & 20.00 & 7.86561 & 5.20996 & 561.52 & $\begin{array}{l}32.72 \\
30.62 \\
31.52 \\
\end{array}$ & 31.620 & IdoEkiti 1 & IdoOsi \\
\hline 10 & 17.80 & 7.86282 & 5.20712 & 588.52 & $\begin{array}{l}42.72 \\
49.12 \\
45.52 \\
\end{array}$ & 45.787 & FMC IdoEkiti & IdoOsi \\
\hline 11 & 22.00 & 7.88018 & 5.19668 & 564.22 & $\begin{array}{l}25.32 \\
22.72 \\
22.92\end{array}$ & 23.653 & Ido 3 & IdoOsi \\
\hline 12 & 23.32 & 7.89160 & 5.19353 & 595.52 & $\begin{array}{l}33.52 \\
31.52 \\
32.52 \\
\end{array}$ & 32.520 & Usi & IdoOsi \\
\hline 13 & 24.70 & 7.90385 & 5.19096 & 554.52 & $\begin{array}{l}28.72 \\
28.52 \\
27.92 \\
\end{array}$ & 28.287 & Usi 2 & IdoOsi \\
\hline 14 & 30.00 & 7.94513 & 5.16533 & 567.22 & $\begin{array}{l}16.02 \\
16.42 \\
16.32 \\
\end{array}$ & 16.253 & Ayetoro & IdoOsi \\
\hline 15 & 36.40 & 8.00161 & 5.15267 & 602.62 & $\begin{array}{l}37.52 \\
36.22 \\
36.52 \\
\end{array}$ & 37.753 & Moba Gram. Otun & Moba \\
\hline 16 & 37.40 & 8.00747 & 5.14237 & 548.12 & $\begin{array}{l}16.62 \\
15.92 \\
16.12 \\
\end{array}$ & 16.220 & 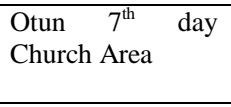 & Moba \\
\hline 17 & 40.04 & 8.02925 & 5.13231 & 559.92 & $\begin{array}{l}20.92 \\
20.52 \\
20.62 \\
\end{array}$ & 20.687 & Otun Extension & Moba \\
\hline 18 & 42.64 & 8.04945 & 5.11995 & 559.52 & $\begin{array}{l}15.22 \\
15.12 \\
15.52\end{array}$ & 15.287 & $\begin{array}{ll}\text { Obaji } & \text { Gram. } \\
\text { OkeEro } & \end{array}$ & Moba \\
\hline 19 & 35.57 & 8.00178 & 5.17614 & 560.62 & 19.02 & 17.987 & IgogoEkiti & \\
\hline
\end{tabular}


Studies on Electric field strength distribution of uhf television signal propagation in Ekiti State. Nigeria.

\begin{tabular}{|c|c|c|c|c|c|c|c|c|}
\hline & & & & & $\begin{array}{l}16.42 \\
18.52\end{array}$ & & & Moba \\
\hline 20 & 35.48 & 8.00354 & 5.18556 & 559.22 & $\begin{array}{l}17.22 \\
16.92 \\
16.52\end{array}$ & 16.877 & Igogo 2 Ekiti & Moba \\
\hline 21 & 36.27 & 8.01350 & 5.19689 & 545.32 & $\begin{array}{l}15.22 \\
16.12 \\
15.82\end{array}$ & 15.720 & IkosuEkiti & Moba \\
\hline 22 & 35.92 & 8.01445 & 5.22030 & 535.32 & $\begin{array}{l}16.22 \\
16.52 \\
16.12\end{array}$ & 16.287 & IkunEkiti & Moba \\
\hline 23 & 31.65 & 7.97809 & 5.26189 & 538.82 & $\begin{array}{l}19.02 \\
20.32 \\
20.52\end{array}$ & 19.953 & IjesamoduEkiti & Ilejemeje \\
\hline 24 & 31.23 & 7.97520 & 5.25004 & 554.22 & $\begin{array}{l}17.02 \\
16.52 \\
16.92\end{array}$ & 16.820 & IyeEkiti & Moba \\
\hline 25 & 30.64 & 7.97027 & 5.25835 & 547.22 & $\begin{array}{l}21.72 \\
23.32 \\
22.82\end{array}$ & 22.620 & Iye LGA & Ilejemeje \\
\hline 26 & 28.37 & 7.94050 & 5.33650 & 574.52 & $\begin{array}{l}22.72 \\
20.82 \\
21.52\end{array}$ & 21.687 & IsanEkiti & Oye \\
\hline 27 & 26.26 & 7.91705 & 5.57298 & 603.42 & $\begin{array}{l}39.22 \\
41.62 \\
40.52\end{array}$ & 40.453 & AyedeEkiti & Oye \\
\hline 28 & 24.51 & 7.89851 & 5.35906 & 589.32 & $\begin{array}{l}39.12 \\
37.62 \\
38.52 \\
\end{array}$ & 38.420 & ItajiEkiti & Oye \\
\hline 29 & 17.42 & 7.82118 & 5.36037 & 564.12 & $\begin{array}{l}50.22 \\
49.42 \\
51.52 \\
\end{array}$ & 50.387 & OyeEkiti & Oye \\
\hline 30 & 16.80 & 7.81886 & 5.35412 & 531.52 & $\begin{array}{l}46.22 \\
47.12 \\
46.92\end{array}$ & 46.753 & OyeEkiti 2 & Oye \\
\hline 31 & 16.82 & 7.81325 & 5.31316 & 551.42 & $\begin{array}{l}36.72 \\
36.52 \\
36.92 \\
\end{array}$ & 36.720 & AyegbajuEkiti & Oye \\
\hline
\end{tabular}

Table 5: Route B Result of the Electric Field Strength Measurement of Channel 41 UHF Television Signal (631.25MHz), of the Broadcasting Service of Ekiti State (BSES TV).

\begin{tabular}{|c|c|c|c|c|c|c|c|c|}
\hline $\begin{array}{l}\text { Observati } \\
\text { on }\end{array}$ & $\begin{array}{l}\text { Line r of } \\
\text { sight from } \\
\text { the TX base } \\
(\mathrm{km})\end{array}$ & Lat. $\left({ }^{\circ} \mathbf{N}\right)$ & $\begin{array}{l}\text { Long. } \\
\left({ }^{0} \mathbf{E}\right)\end{array}$ & $\begin{array}{l}\text { Elevation } \\
\text { ASL/AGL } \\
\text { (m) }\end{array}$ & $\begin{array}{l}\text { Multiple } \\
\text { reading } \\
\text { /E/ field } \\
\text { strength } \\
(\mathrm{dB} \mu \mathrm{V}) \\
\end{array}$ & $\begin{array}{l}\text { Average } \\
\text { reading } \\
/ \mathrm{E} / \\
(\mathrm{dB} \mu \mathrm{V})\end{array}$ & $\begin{array}{l}\text { Description of } \\
\text { location/Town }\end{array}$ & $\begin{array}{l}\text { Local } \\
\text { Govt. } \\
\text { Area }\end{array}$ \\
\hline 1 & 0.00 & 7.72582 & 5.23560 & 415.22 & $\begin{array}{l}80.52 \\
81.22 \\
80.32 \\
\end{array}$ & 80.687 & $\begin{array}{ll}\text { Base } & \text { of } \\
\text { transmitting } \\
\text { station }\end{array}$ & Ado \\
\hline 2 & 20.10 & 7.51487 & 5.27425 & 367.52 & $\begin{array}{l}16.12 \\
16.02 \\
15.92 \\
\end{array}$ & 16.020 & Hospital & Ikere \\
\hline 3 & 20.77 & 7.51080 & 5.23912 & 368.52 & $\begin{array}{l}28.32 \\
36.42 \\
24.52\end{array}$ & 29.753 & Idi Isin, Ikere & Ikere \\
\hline 4 & 21.53 & 7.51678 & 5.19143 & 390.62 & $\begin{array}{l}25.12 \\
24.72 \\
24.32 \\
\end{array}$ & 24.720 & Ikere & Ikere \\
\hline 5 & 23.04 & 7.48925 & 5.24702 & 361.52 & $\begin{array}{l}26.92 \\
26.04 \\
25.22\end{array}$ & 26.060 & $\begin{array}{l}\text { Amoye Gram. } \\
\text { Sch. Ikere }\end{array}$ & Ikere \\
\hline 6 & 31.81 & 7.40986 & 5.27986 & 391.42 & $\begin{array}{l}31.42 \\
30.92 \\
30.52 \\
\end{array}$ & 30.953 & IjuItaogbolu & Ikere \\
\hline 7 & 27.35 & 7.52277 & 5.09054 & 378.22 & $\begin{array}{l}16.12 \\
16.42 \\
16.79 \\
\end{array}$ & 16.443 & $\begin{array}{l}\text { Igbaraodo, St., } \\
\text { Catholic Church }\end{array}$ & $\begin{array}{l}\text { Ekiti South } \\
\text { - West }\end{array}$ \\
\hline 8 & 27.80 & 7.52537 & 5.08243 & 383.02 & $\begin{array}{l}22.72 \\
22.52 \\
22.42 \\
\end{array}$ & 22.553 & $\begin{array}{l}\text { Igbaraodo, } \\
\text { Microfinance } \\
\text { area } \\
\end{array}$ & $\begin{array}{l}\text { Ekiti South } \\
\text { - West }\end{array}$ \\
\hline 9 & 29.72 & 7.61019 & 5.01202 & 476.32 & $\begin{array}{l}25.92 \\
24.52\end{array}$ & 23.920 & IkogosiEkiti & Ekiti West \\
\hline
\end{tabular}


Studies on Electric field strength distribution of uhf television signal propagation in Ekiti State. Nigeria.

\begin{tabular}{|c|c|c|c|c|c|c|c|c|}
\hline & & & & & 21.32 & & & \\
\hline 10 & 27.51 & 7.63127 & 5.02617 & 354.92 & $\begin{array}{l}15.32 \\
15.02 \\
15.12\end{array}$ & 15.153 & ErinjiyanEkiti & Ekiti West \\
\hline 11 & 27.41 & 7.63301 & 5.02670 & 465.32 & $\begin{array}{l}15.12 \\
15.52 \\
15.22\end{array}$ & 15.287 & $\begin{array}{l}\text { Olohan's Palace } \\
\text { Erinjiyan }\end{array}$ & Ekiti West \\
\hline 12 & 22.85 & 7.71279 & 5.06071 & 506.62 & $\begin{array}{l}22.02 \\
22.32 \\
20.62\end{array}$ & 21.653 & $\begin{array}{l}\text { Aramoko } \\
\text { (Okeiro) }\end{array}$ & Ekiti West \\
\hline 13 & 23.25 & 7.72662 & 5.05866 & 475.32 & $\begin{array}{l}15.62 \\
15.92 \\
16.22\end{array}$ & 15.920 & Aramoko 2 & Ekiti West \\
\hline 14 & 26.87 & 7.75086 & 5.03015 & 519.52 & $\begin{array}{l}22.52 \\
23.02 \\
24.02\end{array}$ & 23.187 & ErioEkiti & Ekiti West \\
\hline 15 & 33.10 & 7.74658 & 4.97151 & 452.52 & $\begin{array}{l}15.72 \\
15.92 \\
15.62 \\
\end{array}$ & 15.753 & Itawure & Efon \\
\hline 16 & 34.93 & 7.71196 & 4.95069 & 490.02 & $\begin{array}{l}28.82 \\
29.12 \\
29.92\end{array}$ & 29.287 & $\begin{array}{l}\text { EfonAlaaye LGA } \\
\text { Sect. (Valley) }\end{array}$ & Efon \\
\hline 17 & 35.98 & 7.68755 & 4.94103 & 521.82 & $\begin{array}{l}16.22 \\
16.62 \\
16.12\end{array}$ & 16.320 & Efon 3, (Valley) & Efon \\
\hline 18 & 36.23 & 7.68194 & 4.93891 & 533.92 & $\begin{array}{l}16.12 \\
16.42 \\
16.22\end{array}$ & 16.253 & $\begin{array}{l}\text { Efon4, } \\
\text { (Market) }\end{array}$ & Efon \\
\hline 19 & 24.51 & 7.82023 & 5.08432 & 450.72 & $\begin{array}{l}20.02 \\
20.52 \\
21.02 \\
\end{array}$ & 20.520 & $\begin{array}{l}\text { Govt. College } \\
\text { Area, Efon }\end{array}$ & Efon \\
\hline 20 & 25.38 & 7.83663 & 5.68661 & 505.02 & $\begin{array}{l}24.62 \\
23.52 \\
22.80\end{array}$ & 23.647 & $\begin{array}{l}\text { Roundabout, } \\
\text { Ijero }\end{array}$ & Ijero \\
\hline 21 & 13.59 & 7.69077 & 5.14396 & 575.52 & $\begin{array}{l}35.92 \\
34.12 \\
36.52\end{array}$ & 35.520 & IgedeEkiti & $\begin{array}{l}\text { Irepodun/If } \\
\text { elodun }\end{array}$ \\
\hline 22 & 09.98 & 7.41517 & 5.10517 & 578.52 & $\begin{array}{l}48.02 \\
48.52 \\
49.72\end{array}$ & 48.753 & IyinEkiti & $\begin{array}{l}\text { Irepodun/If } \\
\text { elodun }\end{array}$ \\
\hline 23 & 15.12 & 7.70500 & 5.14812 & 450.12 & $\begin{array}{l}35.12 \\
34.92 \\
35.12\end{array}$ & 35.053 & Ilawe & $\begin{array}{l}\text { Ekiti South } \\
\text { West }\end{array}$ \\
\hline 24 & 32.17 & 7.47012 & 5.46521 & 395.02 & $\begin{array}{l}19.51 \\
19.52 \\
19.53\end{array}$ & 19.520 & Ise & IseOrun \\
\hline 25 & 35.22 & 7.42221 & 5.50121 & 380.52 & $\begin{array}{l}16.82 \\
16.82 \\
16.90\end{array}$ & 16.847 & Emure & Emure \\
\hline 26 & 33.02 & 7.67812 & 5.57451 & 396.52 & $\begin{array}{l}16.92 \\
17.52 \\
17.22\end{array}$ & 17.220 & Orun & Gbonyin \\
\hline 27 & 32.52 & 7.79512 & 5.54543 & 525.02 & $\begin{array}{l}20.52 \\
20.52 \\
20.22\end{array}$ & 20.420 & Ikole & Ikole \\
\hline
\end{tabular}

\subsection{Determined Coverage Areas of BSES in Ekiti State using the Old Transmitter Output Power.}

The primary coverage area between $40 \leq / \mathrm{E} / \leq 82.0 \mathrm{~dB} \mu \mathrm{V}$ within the state covers about $18 \%$ of the total area of the state. The towns and villages in this area have access to the optimum coverage of the television station. Some of the towns and villages falling between this grade are mainly in Ado, Irepodun/Ifelodun, IdoOsi, and part of Ikere and Oye Local Government areas. The secondary coverage area between $20 \leq / \mathrm{E} / \leq 39.0 \mathrm{~dB} \mu \mathrm{V}$ is about $28 \%$ of the State. Some of the towns and villages within this area are mainly IdoOsi, Oye, Ikere, Ekiti South West, and Ekiti West LGAs. Others are in part of Ijero and Ikole LGAs. The tertiary or fringe coverage area $15 \leq / \mathrm{E} / \leq 19.0 \mathrm{~dB} \mu \mathrm{V}$ area of coverage is about $29 \%$ of the State. The towns and villages in this area are mainly in Moba, Ilejemeje, Ekiti East, Efon, Isse-Orun, and Emure Local Government Areas. Others are in part of Gboyin, Ekiti West, Ikere and Ido-Osi LGAs. 
Studies on Electric field strength distribution of uhf television signal propagation in Ekiti State. Nigeria.

Table 6. Some of the towns within the primary coverage area and their respective field strength values

\begin{tabular}{|l|l|l|l|}
\hline S/N & TOWN & LGA & Field Strength dB $\mu$ V) \\
\hline 1 & Ado Ekiti & Ado & 80.02 \\
\hline 2 & Ado Ekiti 2 & Ado & 65.02 \\
\hline 3 & IjeluEkiti & Oye & 60.02 \\
\hline 4 & IfakiEkiti & IdoOsi & 45.02 \\
\hline 5 & Ido (FMC Area) & OdoOsi & 45.02 \\
\hline 6 & AyedeEkiti & Oye & 45.02 \\
\hline 7 & OyeEkiti & Oye & 40.02 \\
\hline 8 & AyegbajuEkiti & Irepodun/Ifelodun & 50.02 \\
\hline 9 & IyinEkiti & Irepodun/Ifelodun & 40.02 \\
\hline 10 & IgedeEkiti & 48.80 \\
\hline
\end{tabular}

Table 7. Some of the towns falling within the secondary coverage area and their respective field strength values.

\begin{tabular}{|l|l|l|l|}
\hline S/N & TOWN & Field Strength $(\mathrm{dB} \mu \mathrm{V})$ & Local Govt.Areas \\
\hline 1 & IdoEkiti & 31.62 & IdoOsi \\
\hline 2 & Orin Ekiti & 26.77 & IdoOsi \\
\hline 3 & UsiEkiti & 32.52 & IdoOsi \\
\hline 4 & Moba Gramm Sch. Area, OtunEkiti & 37.75 & Moba \\
\hline 5 & Itaji & 38.42 & Oye \\
\hline 6 & IsanEkiti & 21.72 & Oye \\
\hline 7 & Ikere (College area) & 24.72 & Ikere \\
\hline 8 & IlaweEkiti & 35.02 & Ekiti West \\
\hline 9 & IgbaraOdoEkiti & 23.55 & Ekiti West \\
\hline 10 & IkogosiEkiti & 23.92 & Ekiti West \\
\hline 11 & Aramoko & 21.65 & Ekiti West \\
\hline 12 & Erio & 23.20 & Ekiti West \\
\hline 13 & Ijero & 22.02 & Ijero \\
\hline
\end{tabular}

Table 8. Some of the towns falling within the Tertiary Coverage area of the station and their respective field strength value

\begin{tabular}{|l|l|l|l|}
\hline $\mathrm{S} / \mathrm{N}$ & TOWN & Field Strength $(\mathrm{dB} \mu \mathrm{V})$ & Local Govt. Areas \\
\hline 1 & Ayetoro & 16.23 & IdoOsi \\
\hline 2 & OtunEkiti & 16.22 & Moba \\
\hline 3 & IgogoEkiti & 18.00 & Moba \\
\hline 4 & IkosuEkiti & 15.72 & Moba \\
\hline 5 & IkunEkiti & 16.30 & Moba \\
\hline 6 & Ijesamodu & 19.95 & Ilejemeje \\
\hline 7 & IyeEkiti & 16.82 & Ilejemeje \\
\hline 8 & Ikere (area) & 16.02 & Ikere \\
\hline 9 & ErinjiyanEkiti & 15.32 & Ekiti West \\
\hline 10 & Aramoko & 15.92 & Ekiti West \\
\hline 11 & Itawure & 15.75 & Efon \\
\hline 12 & EfonAlaaye & 16.32 & Efon \\
\hline 13 & Ise & 19.52 & IseOrun \\
\hline 14 & Emure & 16.82 & Emure \\
\hline
\end{tabular}

Table 9: Table of values for the Ado - Efon Axis.

\begin{tabular}{|l|l|l|l|l|}
\hline S/N & Location & LOS $(\mathrm{km})$ & Field Strength dB $\mu \mathrm{V}$ & Elevation $(\mathrm{m})$ \\
\hline 1 & Tx base Ado/IlokunQrts & 0.00 & 80.67 & 393.92 \\
\hline 2 & IyinEkiti & 9.98 & 48.75 & 578.52 \\
\hline 3 & IgedeEkiti & 13.59 & 36.19 & 575.52 \\
\hline 4 & Aramoko & 22.85 & 21.67 & 506.62 \\
\hline 5 & Erio & 26.87 & 23.19 & 519.52 \\
\hline 6 & Itawure & 33.10 & 15.75 & 452.52 \\
\hline 7 & Efon I & 34.93 & 16.32 & 521.82 \\
\hline 8 & Efon II & 35.98 & 16.25 & 533.92 \\
\hline
\end{tabular}

The deductions from the signal's propagation curve shows that signal strength decreases as distance (LOS) from the transmitter increases in all the routes. This justifies the inverse square law. The few locations with high elevation recorded higher signal strength than expected, thus confirming that high elevation enhances UHF transmission and reception. Propagation curves for all the axes show that; the signal strength of this station was good enough between $0-15 \mathrm{~km}$ radius from the transmitter (above $30 \mathrm{~dB} \mu \mathrm{V}$ ). Beyond $15 \mathrm{~km}$ radius from the transmitter for all routes, signal level was fair (below $30 \mathrm{~dB} \mu \mathrm{V}$ ). 
Table 10: Table of Values for the Ado - Otun Axis.

\begin{tabular}{|l|l|l|l|l|}
\hline $\mathrm{S} / \mathrm{N}$ & Location & LOS $(\mathrm{km})$ & Field Strength $\mathrm{dB} \mu \mathrm{V}$ & Elevation $(\mathrm{m})$ \\
\hline 1 & TX Base & 0.00 & 80.67 & 393.92 \\
\hline 2 & Iworoko & 6.38 & 69.05 & 432.12 \\
\hline 3 & Ifaki & 14.02 & 34.92 & 574.42 \\
\hline 4 & Ido & 20.02 & 31.62 & 562.52 \\
\hline 5 & Usi & 24.72 & 28.30 & 554.52 \\
\hline 6 & Ayetoro & 30.02 & 16.25 & 567.22 \\
\hline 7 & Otun I & 36.42 & 37.75 & 602.62 \\
\hline 8 & Otun II & 40.06 & 20.69 & 559.92 \\
\hline
\end{tabular}

Table 11: Table of values for Ado - Ikere Axis.

\begin{tabular}{|l|l|l|l|l|}
\hline S/N & Location & LOS $(\mathrm{km})$ & Field Strength dB $\mu \mathrm{V}$ & Elevation $(\mathrm{m})$ \\
\hline 1 & TX Base & 0.00 & 80.67 & 393.92 \\
\hline 2 & Ikere & 20.10 & 16.02 & 367.52 \\
\hline 3 & Ikere (Idi Ishin Area) & 20.77 & 26.42 & 368.52 \\
\hline 4 & Ikere: Amoye Gram. Schl Area & 23.04 & 26.04 & 361.52 \\
\hline 5 & Iju/Itaogbolu Police Station Area & 31.82 & 30.97 & 391.42 \\
\hline
\end{tabular}

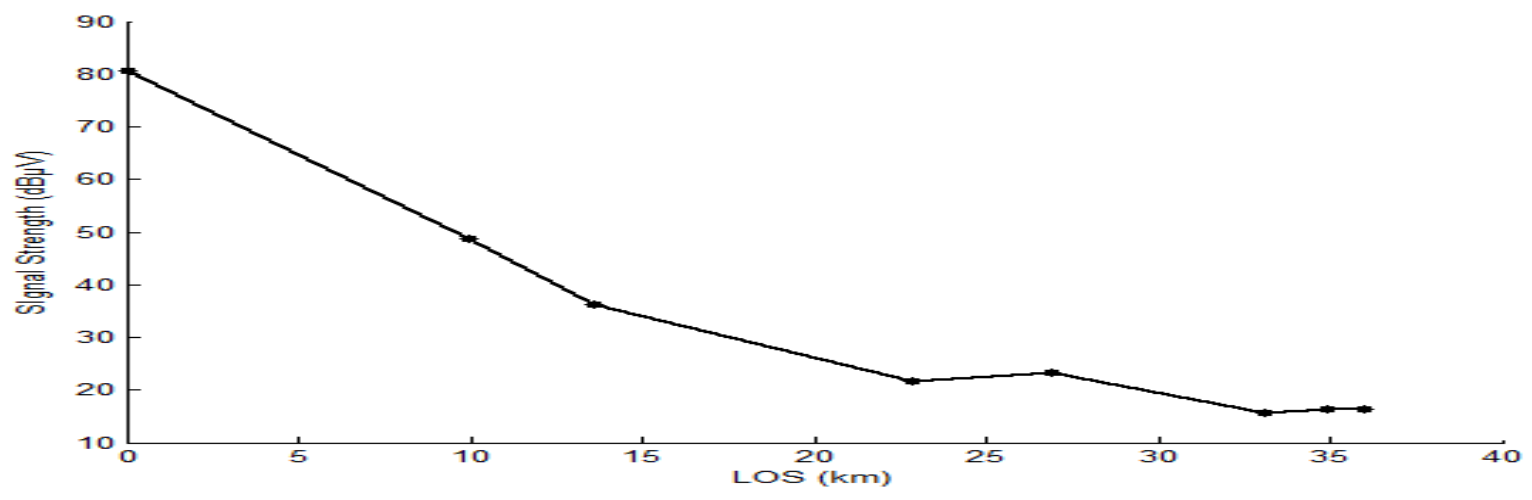

Figure 1: Signal Propagation Curve for Ado - EfonEkiti Axis

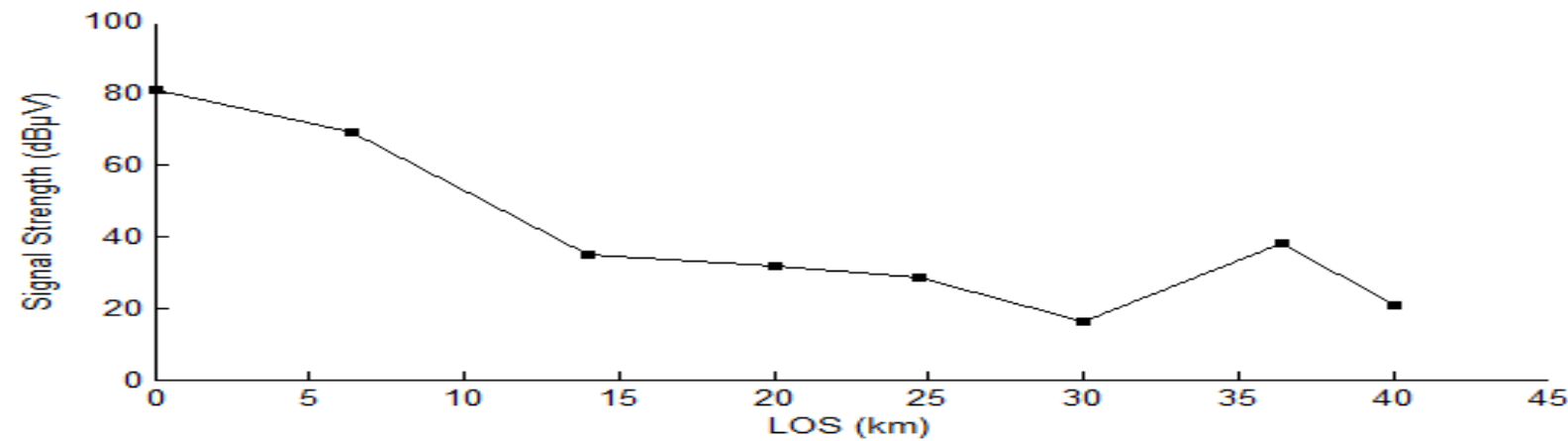

Figure 2: Signal Propagation Curve for Ado - OtunEkiti Axis

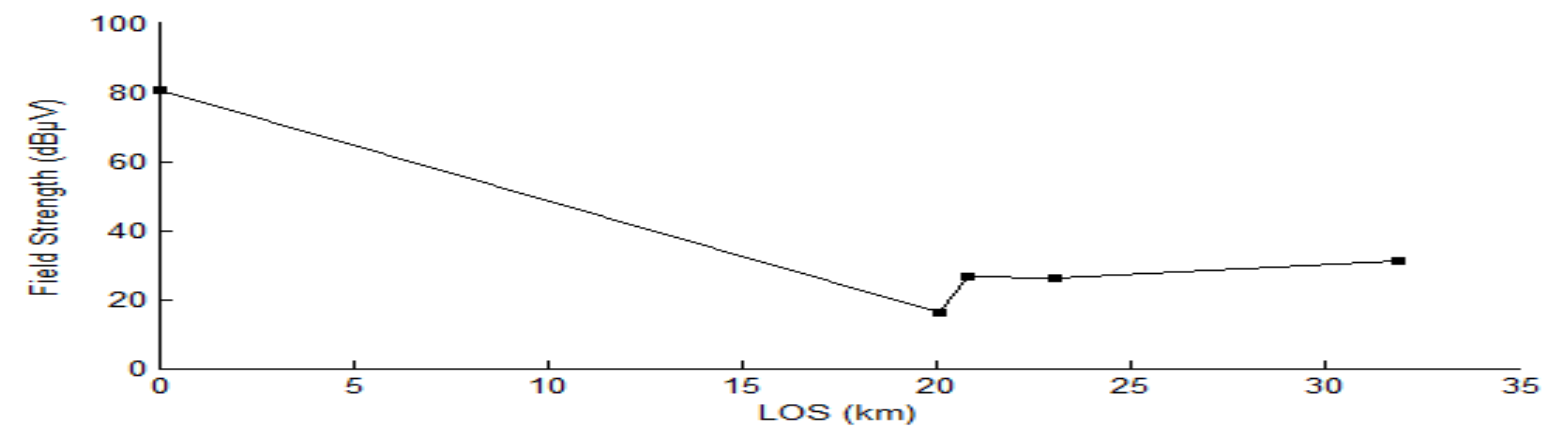

Figure 3: Signal Propagation Curve for Ado - IkereEkiti Axis. 
Table 12: Table of values for Ado - Ikole Axis.

\begin{tabular}{|l|l|l|l|l|}
\hline $\mathrm{S} / \mathrm{N}$ & Location & LOS $(\mathrm{km})$ & Field Strength dB $\mu \mathrm{V}$ & Elevation $(\mathrm{m})$ \\
\hline 1 & Ilokun TX Base & 0.00 & 80.02 & 393.92 \\
\hline 2 & IworokoEkiti & 6.38 & 69.05 & 432.12 \\
\hline 3 & Ifaki & 12.63 & 47.32 & 574.42 \\
\hline 4 & Ayegbaju & 16.84 & 36.72 & 551.42 \\
\hline 5 & Oye & 16.82 & 46.72 & 564.12 \\
\hline 6 & Itapa & 17.52 & 30.02 & 558.02 \\
\hline 7 & Ikole & 30.12 & 17.22 & 560.02 \\
\hline
\end{tabular}

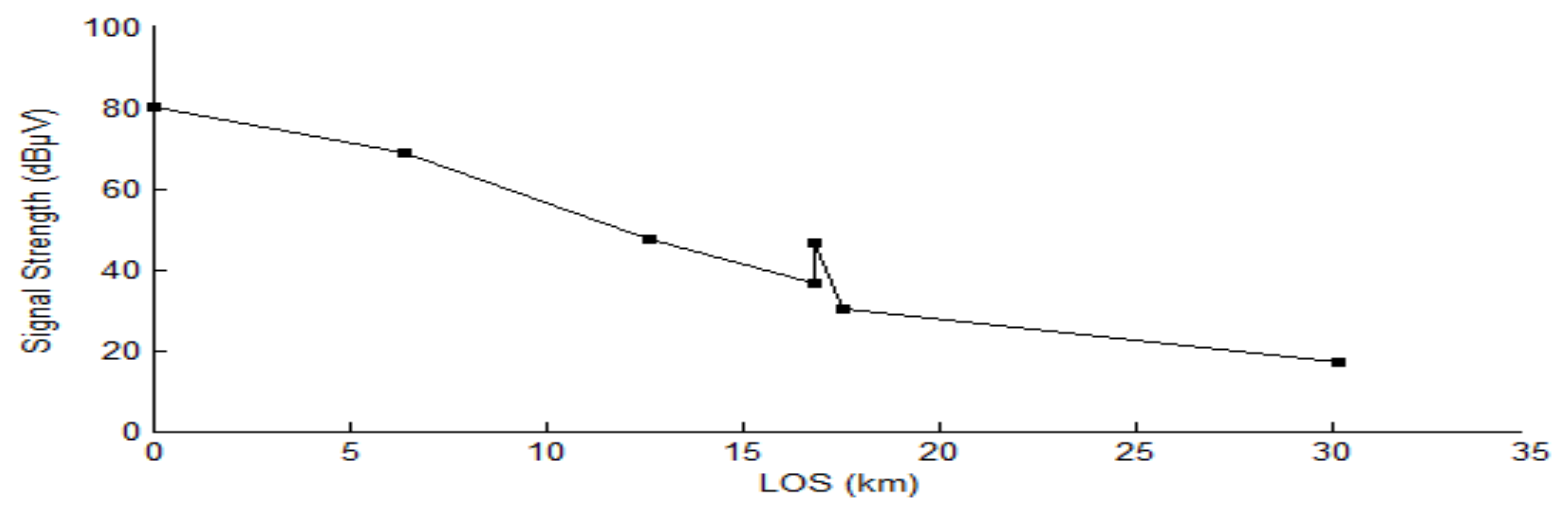

Figure 4: Signal Propagation Curve for Ado - IkoleEkiti Axis.

\subsection{Result of the second phase of measurement}

The table below shows the result of the electric field strength survey in the eight sampled local government areas of Ado, Irepodun/Ifelodun, Ikere, Ekiti South West, Ekiti West, Efon Alaaye, Ijero, and Ido Osi.

\subsection{Comparison of the coverage area of the old transmitter to the new transmitter.}

The comparison of the signal strength of the old transmitting system used till August 22, 2010 by the station to the new system in operation since February 1, 2011 was done. This was carried out using eight local government areas (50\% of the total local government areas) where the electric field strength measurements of the signal were repeated using the new $20 \mathrm{~kW}$ transmitted power as reference. The eight local government areas used for the comparison are; Ado, Ikere, Ekiti West and Irepodun/Ifelodun. Others are Efon, Ekiti South West, Ijero and IdoOsi. Table 4 shows the results of the measurement while table 5 shows the comparison table.

Table 13: Measurement of the Electric Field Strength of the UHF ch.41 Television Signal (BSES) in Ekiti State with the newly installed transmitter power of 20kW.Data Collected in July 2011.

\begin{tabular}{|c|c|c|c|c|c|c|c|c|}
\hline $\mathbf{S} / \mathbf{N}$ & LOS (km) & Lat $\left({ }^{\circ} \mathbf{N}\right)$ & Long $\left({ }^{\circ} \mathbf{E}\right)$ & $\begin{array}{l}\text { Elevation } \\
\text { (m) }\end{array}$ & $\begin{array}{l}\text { Field } \\
\text { Strength } \\
(\mathrm{dB} \mu \mathrm{V})\end{array}$ & $\begin{array}{l}\text { Average } \\
\text { reading } \\
\text { /E/db } \mu \mathrm{V})\end{array}$ & $\begin{array}{l}\text { Description of } \\
\text { Location/Town }\end{array}$ & $\begin{array}{l}\text { Local } \\
\text { Government. } \\
\text { Area }\end{array}$ \\
\hline 1 & 0.00 & 7.7258 & 5.2356 & 393.92 & $\begin{array}{l}95.02 \\
95.52 \\
96.02\end{array}$ & 95.520 & $\begin{array}{l}\text { Base of tx at } \\
\text { Ilokun }\end{array}$ & Ado \\
\hline 2 & 5.05 & 7.7385 & 5.2795 & 409.12 & $\begin{array}{l}80.22 \\
82.12 \\
80.02\end{array}$ & 80.787 & UNAD Gate & Ado \\
\hline 3 & 10.02 & 7.4152 & 5.1152 & 578.22 & $\begin{array}{l}60.52 \\
60.02 \\
61.52\end{array}$ & 60.687 & IyinEkiti & Irepodun/Ifelodun \\
\hline 4 & 13.59 & 7.6908 & 5.1440 & 575.52 & $\begin{array}{l}58.02 \\
57.02 \\
57.52\end{array}$ & 57.520 & $\begin{array}{l}1^{\text {st }} \text { Round about, } \\
\text { Igede }\end{array}$ & Irepodun/Ifelodun \\
\hline 5 & 20.07 & 7.5149 & 5.2743 & 367.52 & $\begin{array}{l}32.52 \\
31.02 \\
32.02\end{array}$ & 31.853 & Hospital & Ikere \\
\hline 6 & 23.27 & 7.7266 & 5.0587 & 475.32 & $\begin{array}{l}30.02 \\
31.02 \\
32.02\end{array}$ & 31.020 & Aramoko & Ekiti West \\
\hline 7 & 26.84 & 7.7508 & 5.0302 & 519.32 & $\begin{array}{l}28.02 \\
27.52 \\
28.22\end{array}$ & 27.920 & ErioEkiti & Ekiti West \\
\hline
\end{tabular}


Studies on Electric field strength distribution of uhf television signal propagation in Ekiti State. Nigeria.

\begin{tabular}{|c|c|c|c|c|c|c|c|c|}
\hline 8 & 27.37 & 7.7508 & 5.0267 & 465.02 & $\begin{array}{l}25.02 \\
24.52 \\
25.12\end{array}$ & 24.887 & Erinjiyan & Ekiti West“ \\
\hline 9 & 33.12 & 7.2850 & 4.7715 & 452.32 & $\begin{array}{l}21.52 \\
20.02 \\
20.52\end{array}$ & 20.687 & Itawure & Efon \\
\hline 10 & 35.02 & 7.7120 & 4.9509 & 490.02 & $\begin{array}{l}20.02 \\
21.02 \\
21.52\end{array}$ & 20.853 & $\begin{array}{l}\text { EfonAlaaye } \\
\text { LGA } \\
\text { Secretariat. }\end{array}$ & Efon \\
\hline 11 & 35.22 & 7.7201 & 4.9500 & 520.52 & $\begin{array}{l}41.02 \\
42.02 \\
41.52 \\
\end{array}$ & 41.520 & $\begin{array}{l}\text { Opposite High } \\
\text { Court, } \\
\text { EfonAlaaye. }\end{array}$ & Efon \\
\hline 12 & 24.52 & 7.8200 & 5.0843 & 450.52 & $\begin{array}{l}33.02 \\
32.52 \\
32.02\end{array}$ & 32.520 & $\begin{array}{l}\text { Roundabout } \\
\text { Area Ijero }\end{array}$ & Ijero \\
\hline 13 & 25.37 & 7.8366 & 5.0867 & 505.02 & $\begin{array}{l}40.02 \\
41.02 \\
41.52\end{array}$ & 40.853 & $\begin{array}{l}\text { Palace Area, } \\
\text { Ijero }\end{array}$ & Ijero \\
\hline 14 & 20.02 & 7.8656 & 5.2100 & 502.52 & $\begin{array}{l}42.02 \\
42.52 \\
43.52\end{array}$ & 42.700 & IdoEkiti & Ido/Osi \\
\hline
\end{tabular}

\subsection{Observation/Deductions from the propagation curve.}

The newly installed transmitter with a transmitted power of $20 \mathrm{~kW}$ gave a better coverage of about $96 \%$ of the state, compared to the old transmitter power of $8 \mathrm{~kW}$ that gave about $75 \%$ coverage of the state. This was deduced using the measurement tables and the propagation curve. High altitudes influence UHF transmission and reception. Father locations with high altitudes recorded high signal values than expected.Terrestrial factors such as Hills, Mountains, trees on the path of UHF signal cause attenuation in the signal strength.

\section{Conclusion and Recommendation.}

4.1. Conclusion: The coverage area of the Broadcasting Service of Ekiti State (BSES) as determined with the old transmitter as reference was about $75 \%$ of the entire landmass of the State. Areas within the optimum coverage $(40 \mathrm{~dB} \mu$ Vand above) are about $18 \%$ of the State, with some of the towns within Ado, Irepodun/Ifelodun, Ido/Osi, Ekiti South West, Ekiti West, Oye, and Ikere Local Government Areas. About 27\% of the State was under secondary coverage i.e. 20-30 dB $\mu \mathrm{V}$, with towns and villages within Ekiti South West, Ekiti West, Ijero, Ikere, Ido-Osi and Oye, Gbonyin, Ikole, Ise Orun local government areas. About $30 \%$ of the State was fairly serviced(15-19 $\mathrm{dB} \mu \mathrm{V}$, tertiary coverage), and the towns and villages falling within this area are mainly in Ise/Orun, Emure, Gbonyin, Ekiti East, Efon, Moba LGAs and part of Ikole, Oye LGAs. About 25\% of the State was not serviced by the station and the towns and villages falling within this area are mainly in the far end of Ikole, Ekiti East, Oye, Ijero and Moba; and few areas within Efon Alaaye, Erinjiyan, Ikere local government areas because of their topographical features. With the new transmitter with $20 \mathrm{kw}$ power output, the result for the eight sampled local government areas showed an enhanced average signal strength of about $40 \%$ in about $0-20 \mathrm{~km}$ line of sight from the station and about $35 \%$ in about $20-40 \mathrm{~km}$ line of sight from the transmitter base, which justifies the investment on the station by the upgraded facilities. About $4 \%$ of the State was not serviced by the station. The towns and villages falling within this area are mainly in the far end of Ikole, Ekiti East, Oye, Ijero, and Moba Local Government areas. Others are in few areas within Efon Alaaye, Erinjiyan, Ikere, etc, because of their topological features. The new station has increased the optimum coverage of the station within the State from about $20 \%$ to about $50 \%$. The signal of the station is now highly dependable in $50 \%$ of the entire land mass. The new coverage area of the station is about $96 \%$ of the entire landmass of the State. However, for more than $50 \%$ of the populace to enjoy the optimum coverage of the station, $(40 \mathrm{~dB} \mu \mathrm{V}$ and above) more effort will still be needed by the management of the Station.

4.2. Recommendation : For further socio-economic development of the people of Ekiti, the following recommendations are made;

i. The State government should endeavour to site booster stations across the State to achieve about $75 \%$ optimum coverage (at lease $40 \mathrm{dBuV}$ ) signal level in $75 \%$ of the entire landmass of the state. This is necessary to ensure that government policies and programmes are well disseminated. These booster stations will be needed in the following local government areas of the State, where the signal strength recorded had become weak. i.e. Efon-Ekiti West areas, Moba - Ilejemeje Areas, Ikole - Ekiti East Areas, Ikere-Ise Orun Areas ii. The transmitter to be installed in any of these routes could be of $1.0 \mathrm{~kW}$ or $1.5 \mathrm{~kW}$ output capacity. This will increase the optimum coverage of the station and not necessarilty cause interference to any neighbouring television stations. It will equally empower the people economically by reducing the money spent on getting active antenna which do not last because they are easilydestroyed by rain and wind. 
iii. The BSES management should endeavor to educate the populace through their channels on the need to make use of active antenna for proper reception at any location beyond $15 \mathrm{~km}$ radials from the base station.

\section{References}

[1] http/www.nbc.gov.com; visited on 12-01-2013

[2] Broadcasting Organization of Nigeria (BON) Directory. 2010, 10-70

[3] G.O. Ajayi, and I.E. Owolabi, "Medium Wave Propagation Curves (for use in medium Wave transmission planning and design)" Technical Report of the Radio Wave Propagation Research Group, Department of Electronic and Electrical Engineering, University of Ife, Nigeria. 1975

[4] G.O. Ajayi, and I.E. Owolabi, Coverage Area of the 10kW, $702 \mathrm{kHz}$ Medium Wave Transmitter at Minna and Feasibility Studies for full Radio Coverage Area of Niger State, Technical Report of the Electrical Communication Consultancy Unit (ECCU), Department of Electrical and Electronic Engineering, University of Ife, Nigeria. 1979

[5] L. Bothias, (1987); Radio Wave Propagation, McGraw-Hill Inc. New York St. Louis San Francisca Montreal Toronto 1987, 144175.

[6] Beasley and Miller (2010): Modern Electronic Communication, $8^{\text {th }}$ edition, Prentice Hall of India pvt. Ltd.2010

[7] G. Kennedy, and D. Bernand, Electronic Communication Systems, McGraw Hill/Macmillan, Singapore, 1992, 80-150

[8] G.O. Ajayi, and I.E. Owolabi, Ground Conductivity Survey in some parts of Nigeria using Radio Wave Attenuation Technique, NJECT, 6(4), 1981, 20-40

[9] CCIR Report 239-6,Propagation Statistics required for Broadcasting Service using the frequency Range 30 to $1000 \mathrm{MHz}$, Recommendations and Reports of the CCIR, Vol 5, ITU, Geneva, In Hall M. (Ed); Ibid, 1986, 256.

[10] R.E Collin, Antennas and Radiowave propagation, McGraw Hill Inc. New Delhi, 1985, 40-409

[11] M. Eiche,Measurement of the electric field strength to determine the Coverage Area of FM Radio Stations in Niger State, Nigeria. 2010

[12] ITU-R, Document 3/11 I-E, Working Party 3J, Draft Handbook on Radio meterology, 1995, 25-60

[13] http/www.en.wikipedia org.

[14] http/www/gps.go/systems/gps/indexhtml 